\title{
A COVID clash: How to proceed when IACUCs and IBCs disagree?
}

\author{
t wasn't easy for Dr. Marty Mayfield \\ to secure a promise from a friend and \\ colleague to send him a small number of \\ mice that exhibited clinical disease when \\ infected with the coronavirus causing \\ COVID-19 disease ${ }^{1}$. The colleague, \\ Dr. David Raush, had been a lab-mate of \\ Mayfield's while they were post-doctoral \\ scholars at Great Eastern University, where \\ Mayfield still worked. The biggest hurdle \\ had been assuring the safe transport of the \\ uninfected animals to Great Eastern because \\ an escaped mouse, even if not carrying \\ the virus, would be a public health threat. \\ Although that problem was resolved, an \\ equally significant problem faced Mayfield. \\ Mayfield proposed to perform his mouse \\ experiments at the ABSL-3 level and keep \\ the SARS-CoV-2 coronavirus under BSL-3 \\ conditions. But although Mayfield had proper \\ past experience working under BSL-3 and \\ ABSL-3 conditions, Great Eastern did not \\ have BSL- 3 or ABSL-3 facilities. Mayfield
}

was well aware of this problem and with the knowledge of the school's IACUC and Institutional Biosafety Committee (IBC) chairpersons, he had submitted IACUC and IBC applications to a nearby contract research organization (CRO) that had the needed biocontainment facilities and would allow Mayfield to use those facilities. Both of those applications had been approved by the CRO and now, following Great Eastern policy, the same approved applications were submitted for concurrence by the IBC and IACUC of Great Eastern University. The IACUC voted to agree with the CRO's approval, but the IBC did not, citing inadequate containment if a mouse were to escape from its cage while at the CRO. The result was a de facto halt to the planned experiments.

Mayfield was livid, and after an informational phone call to OLAW he argued with the IACUC chairman that only one IACUC was legally required to approve a protocol application, and he already had that approval from the CRO's IACUC. There was no need for any additional concurrence by the Great Eastern IBC, said Mayfield, especially after the Great Eastern IACUC agreed with the CRO's IACUC and the CRO's IACUC was in agreement with the CRO's biosafety committee. The chairman brought the issue to the Great Eastern IACUC. What are the pertinent regulatory requirements for the Great Eastern IACUC to follow and would you support Mayfield or the Great Eastern IACUC?

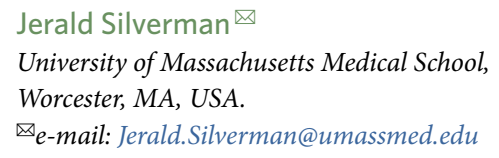

Published online: 18 December 2020 https://doi.org/10.1038/s41684-020-00686-8

References

1. Dinnon, K. H. III et al. Nature 586, 560-566, https://doi.org/ 10.1038/s41586-020-2708-8 (2020).

\section{Compliance requires communication and cooperation}

$\mathrm{T}$ his scenario could have been avoided with better communication and planning. Although Great Eastern University (GEU) has an institutional policy that requires concurrence with collaborating entity oversight committees, guidance on how conflicts are resolved or negotiated appears lacking. The GEU IACUC should have developed guidelines for collaborative studies with other institutions that included a requirement for a written agreement with the collaborating organization and the responsibilities of each party for the various aspects of the animal care and use program such as protocol review, animal ownership, veterinary care, and IACUC oversight authority ${ }^{1}$. A written collaboration agreement can also specify oversight responsibilities specific to biohazards and IBC review. The Institutional Biosafety Committee (IBC) from each institution should (promptly) communicate to try to allay the concerns of GEU's IBC. Considering the close proximity of the two facilities it should even be possible for the GEU IBC or some of its members to visit the Contract Research Organization (CRO) to see if their concerns are justified, or to suggest amendments to the CRO IBC application.

Mayfield was correct when he said that approval by more than one IACUC is not required to meet federal regulatory requirements ${ }^{2}$, but GEU has an internal policy that requires concurrence with external review, presumably by all appropriate compliance committees. The GEU IACUC's concurrence with the CRO's IACUC and IBC may not "override" the requirement for their IBC to also concur.

There are a variety of details missing from this scenario that would help determine the best course of action to take. If the project is funded by an agency that has adopted PHS Policy, both institutions may be required to have a PHS Assurance, but GEU must verify IACUC approval. If both institutions are AAALAC International accredited, that could make the process of dual oversight simpler and may help alleviate the concerns of the GEU IBC. In this case, if the details were included in the written understanding, it would seem more appropriate to have the CRO's committees be responsible for the protocol approval because the animal work will take place there. Also, their committees are likely to have more experience working with ABSL-3 studies. GEU should ask for the documents associated with IBC approval for the ABSL-3 work as well as follow-up documents such as annual reviews, any noncompliance, etc.

Institutions should develop policies and procedures that allow them to easily resolve issues that arise when collaborative research projects are presented to them. One institution has implemented and published methods for facilitating regulatory oversight for multi-site research. This "could provide a model for a distributed, national network of IACUC reliance "." 